\title{
Evolutionary Entropy: A Predictor of Body Size, Metabolic Rate and Maximal Life Span
}

\author{
Lloyd Demetrius ${ }^{\mathrm{a}, \mathrm{b}, *}$, Stéphane Legendre ${ }^{\mathrm{c}}$, Peter Harremöes ${ }^{\mathrm{d}}$ \\ ${ }^{a}$ Department of Organismic and Evolutionary Biology, Harvard University, Cambridge, MA, \\ USA \\ ${ }^{b}$ MPI for Molecular Genetics, 14195, Berlin, Germany \\ ${ }^{c}$ Laboratoire Ecologie et Evolution (UMR 7625), Ecole Normale Supérieure, 46 rue d'Ulm, \\ 75230 Paris cedex 05, France \\ ${ }^{d}$ Centrum voor Wiskunde en Informatica (CWI), Kruislaan 413, Amsterdam, The Nederlands
}

Received: 17 July 2008 / Accepted: 25 November 2008 / Published online: 27 January 2009

(c) The Author(s) 2009. This article is published with open access at Springerlink.com

\begin{abstract}
Body size of organisms spans 24 orders of magnitude, and metabolic rate and life span present comparable differences across species. This article shows that this variation can be explained in terms of evolutionary entropy, a statistical parameter which characterizes the robustness of a population, and describes the uncertainty in the age of the mother of a randomly chosen newborn. We show that entropy also has a macroscopic description: It is linearly related to the logarithm of the variables body size, metabolic rate, and life span. Furthermore, entropy characterizes Darwinian fitness, the efficiency with which a population acquires and converts resources into viable offspring. Accordingly, entropy predicts the outcome of natural selection in populations subject to different classes of ecological constraints. This predictive property, when integrated with the macroscopic representation of entropy, is the basis for enormous differences in morphometric and life-history parameters across species.
\end{abstract}

Keywords Evolutionary entropy $\cdot$ Body size $\cdot$ Metabolic rate $\cdot$ Maximal life span · Allometric relations

\section{Introduction}

Body size is a fundamental property of organisms. Size regulates the rate at which an organism transforms the energy of resources into biological work, hence it determines metabolic rate. Size also controls the duration of fundamental physiological events such as the turnover time of metabolic processes. Accordingly, maximal life span, which is an aggregate of physiological and developmental events, is also a function of body size.

\footnotetext{
*Corresponding author.

E-mail address: ldemetr@oeb.harvard.edu (Lloyd Demetrius).
} 
These morphometric and physiological properties are characterized by a large interspecific variation. Body sizes spans 24 orders of magnitude. Metabolic rate and life span exhibit similar variation. Maximal life span, for example, varies 5,000 fold among insects, 100 fold among mammals, and 15 fold among birds (Calder, 1984). There also exist systematic differences in life span between birds and mammals: Birds are much longer lived than mammals of the same body size. Size and life-history variables are also characterized by temporal and spatial ecotypic patterns (Bergmann, 1847; Peters, 1983; Lomolino, 1985). A well-documented trend is Cope's rule, the increase in mean body size within certain phyletic lineages (Cope, 1886), see Bonner (1988; Chap. 2).

This article provides an adaptive explanation of this interspecific variation in terms of the concept evolutionary entropy, an analogue of the Gibbs-Boltzmann entropy in statistical thermodynamics.

Evolutionary entropy is a function of the age-specific fecundity and mortality variables and has an information-theoretic interpretation: the uncertainty in the age of the mother of a randomly chosen newborn (Demetrius, 1974). Entropy characterizes the robustness or stability of a population, the rate at which it returns to its steady state condition after a random perturbation. Entropy also determines Darwinian fitness, the capacity of a rare variant type to displace the resident in competition for the available resources (Demetrius, 1997; Demetrius and Ziehe, 2007).

When resources are limited but constant, there will be little variation in population size. Accordingly, variants with increased entropy - in view of the increased robustness this confers-will be more efficient at acquiring the limited resources. Consequently, such mutants will prevail over the ancestral type. When resources are abundant but variable, mutants with decreased entropy, and a concomitantly decreased robustness, will be more efficient in converting the resources into viable offspring. The variability in resource abundance, however, entails that the invasion dynamics will be characterized by large variations in population size. When the mean population size is large, the selective process will be deterministic and mutants with decreased entropy will dominate. However, when the mean population size is small, stochastic effects will dominate and the selective outcome will be unpredictable.

Selective advantage, as the above description indicates, will depend on both the capacity of an organism to acquire resources-a property which will be regulated by its metabolic rate and the efficiency in converting resources into viable offspring - a condition which will be contingent on the body size of the organism. Efficiency in acquiring and converting resources will also be reflected in individual survivorship, and hence correlated with the organism's life span.

We conjecture from these observations that the statistical measure evolutionary entropy should also characterize macroscopic population variables such as metabolic rate, body size, and life span. Accordingly, these species-specific properties should be predictable from the age-specific fecundity and mortality variables of a population-the quantities which are required to compute the statistical representation of entropy. This article establishes analytic, computational, and empirical support for the relations of evolutionary entropy with the macroscopic variables body size, metabolic rate, and life span.

We first derive an analytic relation between entropy and the generation time, the mean age at which individuals produce offspring. This relation is validated by a computer simulation and empirical study using the life-tables of bird, mammal, and insect species. We then integrate the analytic relation between entropy and generation time with a series of 
allometric relations between life-history variables and body size to derive a new class of macroscopic characterizations of entropy. These relations are illustrated with data on body size, metabolic rate, and maximal life span in bird, mammal, and insect species.

The concept evolutionary entropy is the cornerstone of directionality theory, an analytic model of the evolutionary process which predicts changes in the genotypic and phenotypic composition of populations under various ecological constraints. We discuss the implications of this theory to address problems regarding the large variations in body size, metabolic rate, and life span observed in populations.

\section{Methods}

\subsection{Entropy and generation time}

The dynamics of age-structured populations can be described in terms of the age-specific fecundity and mortality variables, $l(x)$, the chance that a newborn survives to age $x, m(x)$, and the mean number of offspring produced by an individual of age $x$.

A fundamental parameter in this model is the quantity $p(x)$, the probability distribution of the parental age of a randomly chosen newborn individual:

$$
p(x)=e^{-r x} V(x) .
$$

Here, $r$ denotes the population growth rate, the rate of increase in population numbers and $V(x)=l(x) m(x)$ the net-reproductive function.

Evolutionary entropy, as derived in the context of the ergodic theory of population dynamics, is given by

$$
S=-\int_{\alpha}^{\beta} p(x) \log p(x) d x .
$$

Here, $\alpha$ is the age at first reproduction and $\beta$ the age at which reproduction ceases. Hence, $S$ is a function of age of sexual maturity, size of progeny sets, and reproductive span. The quantity $S$ describes the uncertainty in the age of the mother of a randomly chosen newborn (Demetrius, 1974) and characterizes three main demographic properties:

(a) The degree of iteroparity of the population. Semelparous populations where reproduction occurs at a single instant in the life cycle have zero entropy. Iteroparous populations where organisms reproduce at several distinct stages in the life cycle have positive entropy.

(b) The robustness or demographic stability of the population, that is, the rapidity with which the population returns to the steady state condition after a random perturbation in the age-specific birth and death parameters (Demetrius, 1977; Demetrius et al., 2004).

(c) The position of the population along the fast-slow life-history continuum:

- Large entropy: late age at sexual maturity, small net-progeny sets, broad reproductive span.

- Small entropy: early age at sexual maturity, large net-progeny sets, narrow reproductive span. 
Generation time, the mean age of mothers at the birth of their offspring, is given by

$$
T=\int_{\alpha}^{\beta} x p(x) d x .
$$

The quantity $S$ as given by Eq. (1) is a statistical parameter expressed in terms of the microscopic variables, the age-specific fecundity, and mortality rates. We will now show that the entropy $S$ is analytically related to the generation time $T$ :

$$
S \approx \log T+b,
$$

where $b=b(T)$ is a species-specific constant.

We will first give a probabilistic argument in support of Eq. (4) and then provide additional computational support and an empirical analysis using life tables of birds, mammals, and insects populations.

\subsection{The probabilistic argument}

We denote by $X$ the random variable with probability density function $p(x)$ given by Eq. (1). Consider the function $p^{*}(x)$ defined by

$$
p^{*}(x)=\frac{p(x / k)}{k} .
$$

The function $p^{*}(x)$ represents the density function of a related species and $k$ is a constant indicating how the life-histories of the two species scale with each other.

The entropy function $S^{*}$ associated with the density function $p^{*}(x)$ is

$$
\begin{aligned}
S^{*} & =-\int p^{*}(x) \log p^{*}(x) d x \\
& =-\int \frac{p(x / k)}{k} \log \left[\frac{p(x / k)}{k}\right] d x \\
& =-\int \frac{p(x / k)}{k} \log [p(x / k)] d x+\log k \\
& =-\int p(x) \log p(x) d x+\log k .
\end{aligned}
$$

Hence, $S^{*}=S+\log k$. Now

$$
\begin{aligned}
\log \left[E\left(X^{*}\right)\right] & =\log \int x\left[\frac{p(x / k)}{k}\right] d x \\
& =\log k+\log \int x p(x) d x \\
& =\log k+\log [E(X)] .
\end{aligned}
$$

Consequently, the generation time given by $T=E(X)$, Eq. (3), satisfies

$$
\log T^{*}=\log k+\log T .
$$


Hence, we obtain

$$
S-\log T=S^{*}-\log T^{*}=b
$$

where $b$ denotes a taxon specific constant. We now have

$$
S=\log T+b .
$$

The relation given by Eq. (5) will hold for any pair of species that are closely related so that their life-history scales as described above. Accordingly, a plot of $(\log T, S)$ for species within the same taxa should give points on a straight line with slope 1 .

Example. The expression given by Eq. (5) can be illustrated by taking as $p(x)$ the $\log$ normal distribution, namely

$$
p(x)=\frac{1}{x \sigma \sqrt{2 \pi}} \exp \left(-\frac{(\log (x)-\mu)^{2}}{2 \sigma^{2}}\right) .
$$

This distribution, as observed in Smith (2004), provides a good description of the netreproductive function for various natural populations. We assume that $\sigma$ is constant for closely related species and that $\mu$ varies. A rescaling given by $p^{*}(x)=p(x / k) / k$ yields a lognormal distribution.

The generation time $T$ and entropy $S$ associated with the distribution $p(x)$ are given by

$$
\begin{aligned}
& T=\exp \left(\mu+\frac{\sigma^{2}}{2}\right), \\
& S=\mu+\frac{\log \left(2 \pi e \sigma^{2}\right)}{2} .
\end{aligned}
$$

Hence, $\mu=\log T-\sigma^{2} / 2$ and we obtain $S=\log T+b$ with $b=(\log 2 \pi e+$ $\left.\log \sigma^{2}-\sigma^{2}\right) / 2$. We note that $b$ can assume a large range of values contingent on $\sigma^{2}$ : it increases for $\sigma^{2}$ in the interval $[0,1]$, and decreases for $\sigma^{2}$ in the range $1<\sigma^{2}<\infty$.

\subsection{The computational argument}

The probabilistic argument is in terms of the differential entropy, which is the entropy of a continuous random variable. The computational argument we now give is in terms of a discrete random variable and we write $S=-\sum_{j} p_{j} \log p_{j}$. In view of the discretization, the relation between $S$ and $T$ will not be exact as in (5), but approximate.

We provide a Monte Carlo simulation to support Eq. (4), using an extended $2 \times 2$ Leslie matrix

$$
\left[\begin{array}{ll}
\sigma s_{0} f_{1} & \sigma s_{0} f_{2} \\
s_{1} & s_{2}
\end{array}\right],
$$

with primary sex-ratio $\sigma=0.5$, survival rates $s_{0}, s_{1}, s_{2}$, and fecundities $f_{1}, f_{2}$. The demographic parameters are given reference values as in Legendre et al. (1999), and are 


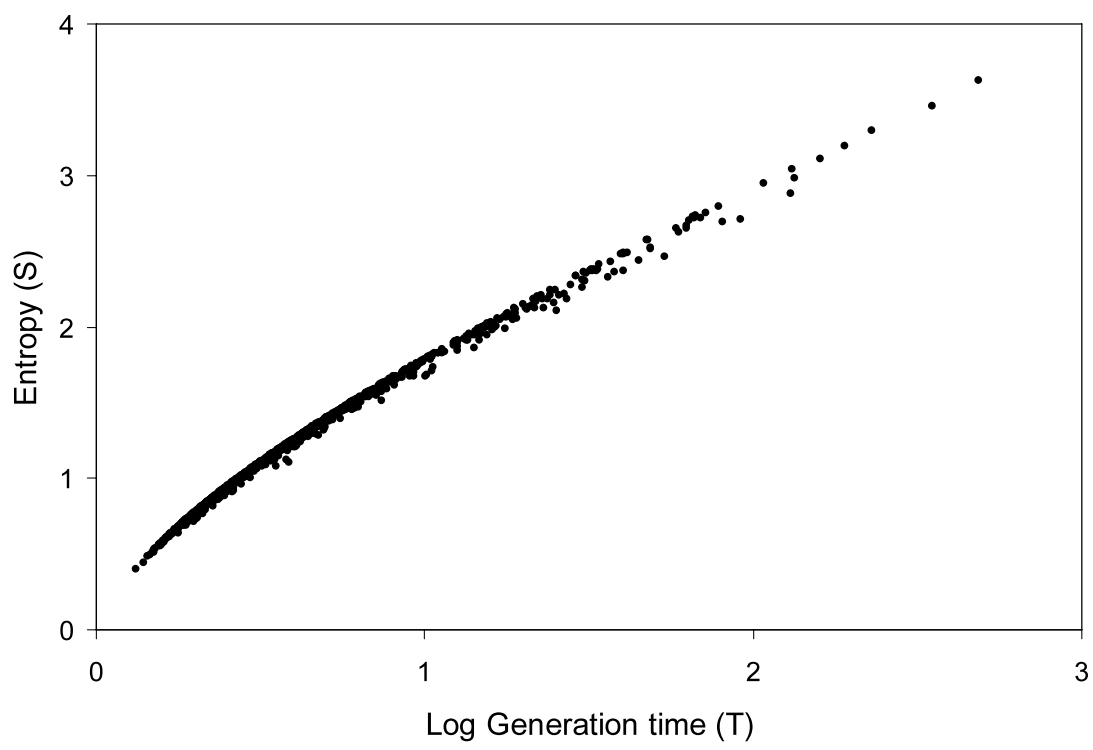

Fig. 1 The relation of logarithm of generation time $(T)$ to demographic entropy $(S)$ for a random extended $2 \times 2$ Leslie matrix (1,000 realizations). Average values of demographic parameters: survival rates $s_{0}=0.2, s_{1}=0.35, s_{2}=0.5$; fecundities $f_{1}=f_{2}=7$.

randomized around their reference values: for survival, to ensure values in $[0,1]$, a beta distribution with standard deviation 0.1 is used; for fecundity, to ensure nonnegative values, a truncated Gaussian distribution with standard deviation 0.1 is used. Fig. 1 shows entropy versus the logarithm of generation time for 1,000 realizations of the random matrix. A linear relationship obtains, as predicted by Eq. (4).

\subsection{Empirical studies}

The empirical study is based on an analysis of several datasets:

(1) Birds: 13 life cycles of bird populations from Niel and Lebreton (2005) (Fig. 2a).

(2) Mammals: 127 life cycles of mammal populations from Oli and Dobson (2003) to which we have added data for Homo sapiens (Fig. 2b).

(3) Insects: 20 life cycles of insect populations comprising 13 species, some at different temperatures (Appendix; Fig. 2c).

In birds, mammals, and insects we observe a good agreement of empirical data with Eq. (4).

\subsection{Body size, metabolic rate and life span: the relation with entropy}

We will now exploit the relation between entropy and generation time to derive a series of analytic rules relating the macroscopic parameters metabolic rate, body size, and maximal life span with entropy. Our derivation is based on a series of allometric relations between body size and other life-history variables. 


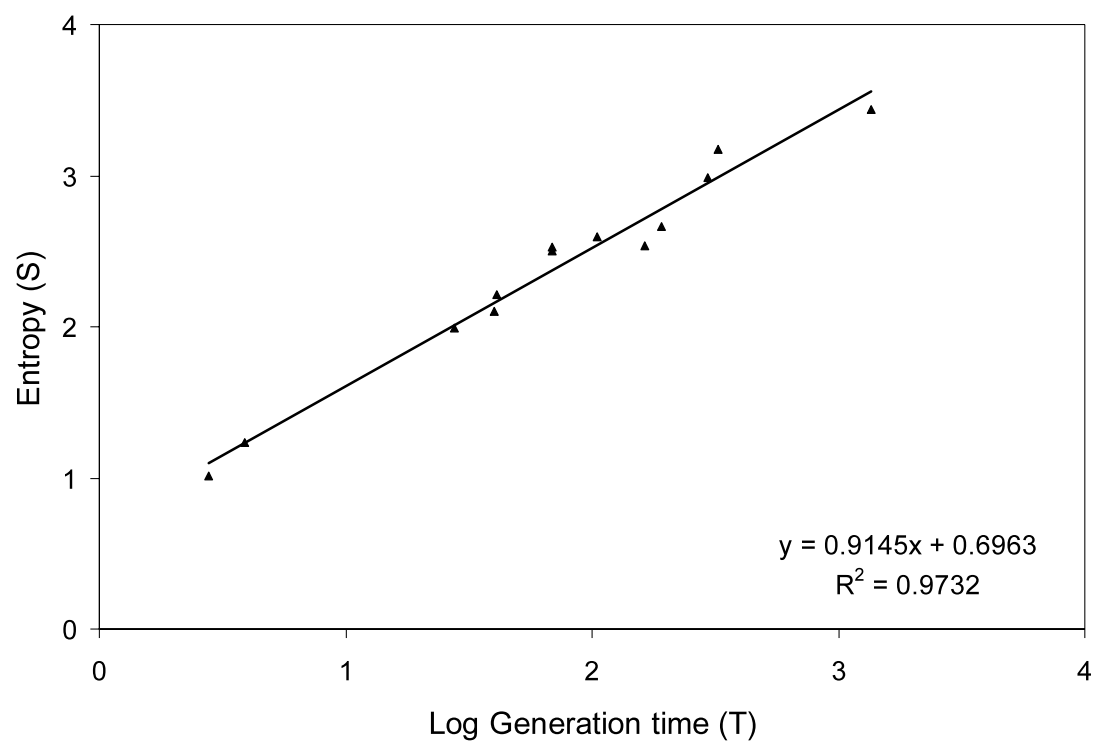

(a)

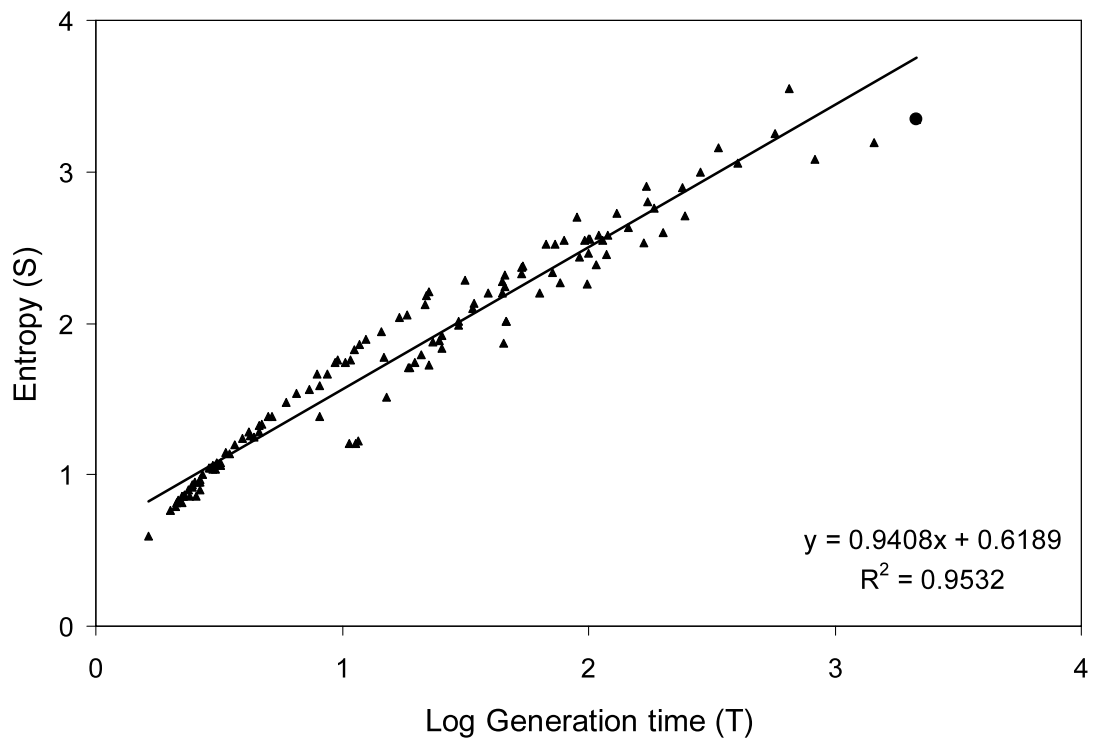

(b)

Fig. 2 The relation of logarithm of generation time $(T)$ to demographic entropy $(S)$ with linear fit. (a) Birds data set. (b) Mammals data set (the large dot stands for Homo sapiens). (c) Insects data set. 


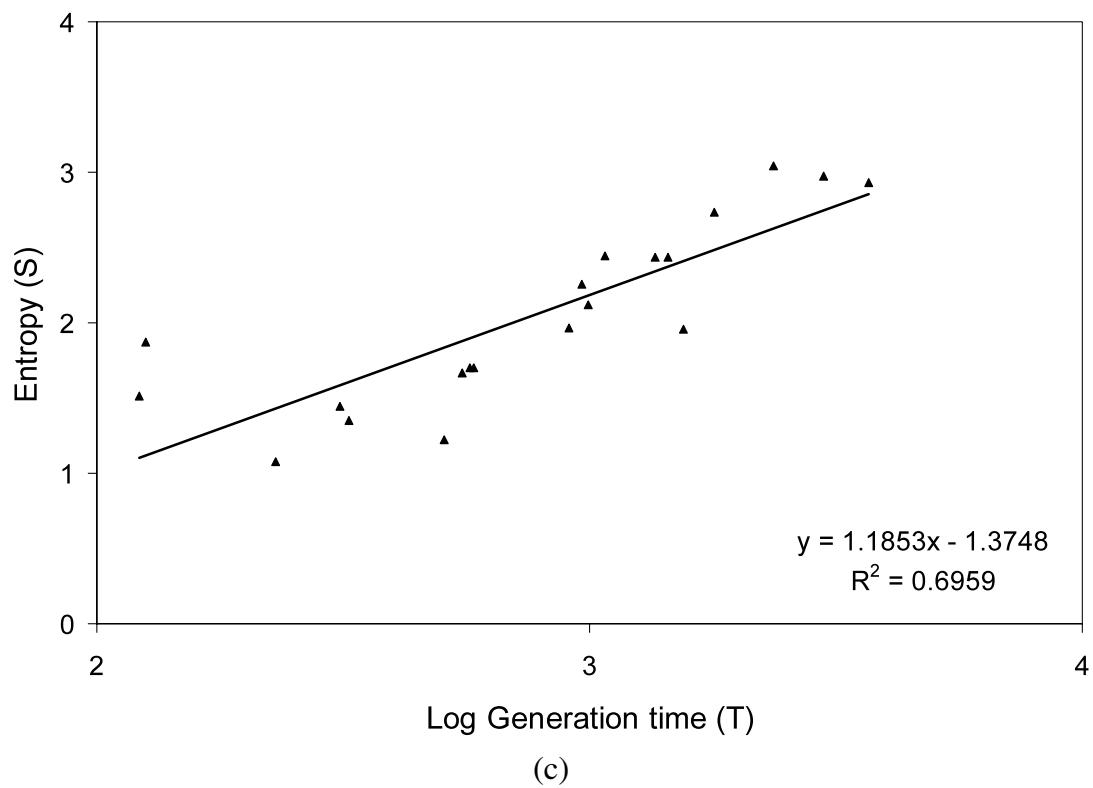

Fig. 2 Continued.

Adult body size underlies all aspects of an organism's physiology. In view of this property and its relative accessibility in empirical studies, the parameter has been the basis for comparing the life-history and physiological properties of different organisms. These studies have led to a large class of empirical rules relating body size with various measures of physiological time and metabolic rate; see Peters (1983), Calder (1984), for example.

In Demetrius (2006), we provided a molecular explanation for these empirical rules. The model recognized that metabolic activity within organisms has its origin in the processes of energy transduction localized in biomembranes. These processes are regulated by the transport of protons across the energy-transducing membranes, and the coupling of two molecular motors - the redox reaction and ADP phosphorylation. We integrated the chemiosmotic theory of energy transduction within biomembranes with the methods of quantum statistics, to study metabolic activity at the cellular level and to relate metabolic rate with cell size. A multi-level scaling argument was then invoked to extend these single-cell results to multi-cellular organisms. We derived the following expression relating metabolic rate $P$ with body size $W$

$$
P=\alpha C \Delta p W^{\beta} \text {. }
$$

The proportionality constant in the allometric relation has three components:

(i) $\alpha$, a measure of metabolic efficiency. It is given by the ratio $\rho_{1} / \rho_{2}$, where $\rho_{1}$ is the rate of reductant supply to the energy-transducing membrane, and $\rho_{2}$ the rate of nutrient uptake by the organism.

(ii) $C$, the proton conductance of the energy-transducing membrane. 
(iii) $\Delta p$, the proton motive force, which describes the free energy stored in the membrane electrochemical proton gradients.

The quantity $\alpha$ depends on the geometry of the pathways of energy flow within the organism. The bioenergetic variables $C$ and $\Delta p$ depend on the physical and chemical composition of the energy-transducing membranes.

The scaling exponent $\beta$ is contingent on the mechanisms - classical or quantized - that regulate the flow of energy within the membrane. A classical mechanism corresponds to the case where the distribution of energy levels is continuous and we have $\beta=1$. A quantized process characterizes the situation where the distribution of energy levels is discrete. In this case, $\beta$ will assume the values $2 / 3$ or $3 / 4$ depending on the degree of quantization of the energy transduction processes.

A more compact representation of Eq. (6) is

$$
P=a_{1} W^{\beta}
$$

The allometric relations for measures of physiological time, the generation time $T$ and the maximal life span $L$, derived by the same methods, have forms analogous to Eq. (6):

$$
\begin{aligned}
& T=a_{2} W^{1-\beta}, \\
& L=a_{3} W^{1-\beta} .
\end{aligned}
$$

We will now appeal to the analytic relation between entropy and generation time, Eq. (4), and to allometric laws expressed by Eqs. (7) to obtain a series of relations between entropy and the macroscopic parameters, body size, metabolic rate, and maximal life span. The relations we derive will have the general form

$$
S=\tilde{a} \log X+\tilde{b}
$$

Here, $\tilde{a}, \tilde{b}$ are taxon-specific constants and $X$ represents a physiological or life-history variable.

The logarithmic dependence is related to the fact that the information theoretic representation for entropy, Eq. (2), is equivalent to the representation

$$
S=\tilde{k} \log M
$$

Here, $M$ denotes the number of typical genealogies-or pathways of energy flowgenerated by the population (Demetrius, 1983).

\section{Results}

\subsection{Entropy and macroscopic parameters}

Entropy and body size. We can conclude from Eqs. (4) and (7b) that

$$
S \approx(1-\beta) \log W+b_{1} .
$$


The empirical support for Eq. (8a) is presented for birds in Fig. 3a, and for mammals in Fig. 3b.

Entropy and metabolic rate. We can infer from Eqs. (4), (7a), and (7b) that

$$
S \approx \frac{1-\beta}{\beta} \log P+b_{2}
$$

The empirical support for Eq. (8b) is presented for birds in Fig. 4a (data from Mc Kechnie and Wolf, 2004), and for mammals in Fig. 4b (data from Savage et al., 2004). The metabolic rate $P$ was only available for a fraction of the species.

Entropy and maximal life span. We conclude from Eqs. (4), (7b), and (7c) that

$$
S \approx \log L+b_{3}
$$

The empirical support for Eq. (8c) is presented for birds, mammals and insects in Fig. 5. For birds and mammals, we used recorded values of $L$ from the AnAge Database (Magalhães et al., 2006). For insects, we used the maximal life span observed in the published life tables.

\subsection{Prediction and empirical results}

The relations (8a), (8b), and (8c) involve the scaling exponent $\beta$. This quantity is not a universal constant, as suggested by the empirical studies of Kleiber (1961). For recent statistical analysis, see Dodds et al. (2001), Glazier (2005), Reich et al. (2006)—indicate that $\beta$ ranges from $2 / 3$ to 1 . Birds are typically described by $\beta=2 / 3$, large mammals by $\beta=3 / 4$ and perennial plants by $\beta=1$. We expect that the slope of the empirical curves will be contingent on the class of organisms which are used in the analysis.

We can appeal to the different scaling exponents $\beta=2 / 3$ for birds, and $\beta=3 / 4$ for mammals to predict the following patterns regarding the regression slopes in Figs. 3 and 4.

(a) In the case of body size, the regression slope $1 / 3$ for birds and $1 / 4$ for mammals.

(b) In the case of metabolic rate, the regression slope $1 / 2$ for birds and $1 / 3$ for mammals.

A study of Figs. 3 and 4 indicates that the empirical data are consistent with the predictions. In the case of birds (Figs. $3 \mathrm{a}$ and $4 \mathrm{a}$ ), the regression slopes are 0.27 and 0.56 , respectively. The predicted values are 0.33 and 0.5 . For mammals (Figs. $3 b$ and $4 b$ ), the regression slopes are 0.14 and 0.18 , respectively. The predicted values are 0.25 and 0.33 . The mammalian data include both large and small organisms, and hence a departure from the scaling exponent $\beta=3 / 4$. This may account for the discrepancy between predicted and observed values in the mammalian data.

\section{Discussion}

Body size, a fundamental physiological and ecological characteristic, regulates the rate at which an organism transforms external energy into biological work. Hence, it determines metabolic rate, the rate at which the chemical energy is generated by cellular metabolic 


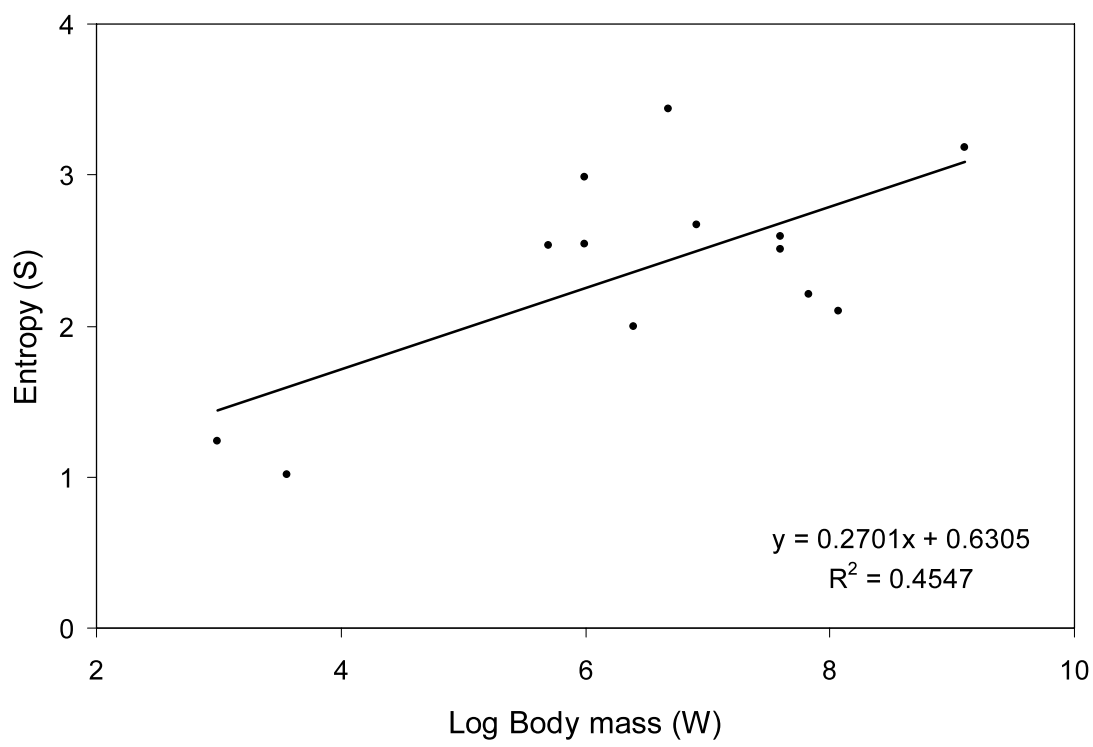

(a)

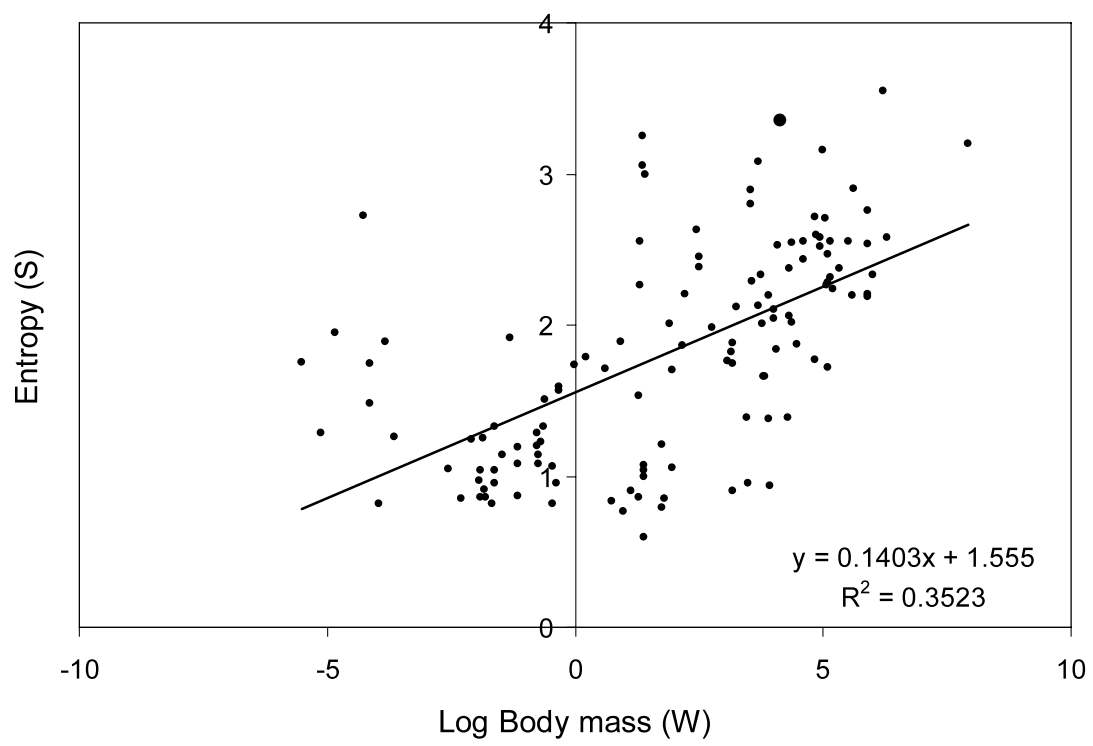

(b)

Fig. 3 The relation of logarithm of body mass $(W)$ to demographic entropy $(S)$ with linear fit. (a) Birds data set ( $W$ in g). (b) Mammals data set (the large dot stands for Homo sapiens) ( $W$ in $\mathrm{kg}$ ).

networks. Size also controls the duration of fundamental physiological events such as the turnover time of metabolic processes. Accordingly, maximal life span, which is an aggregate of physiological and developmental events, is also a function of body size. 


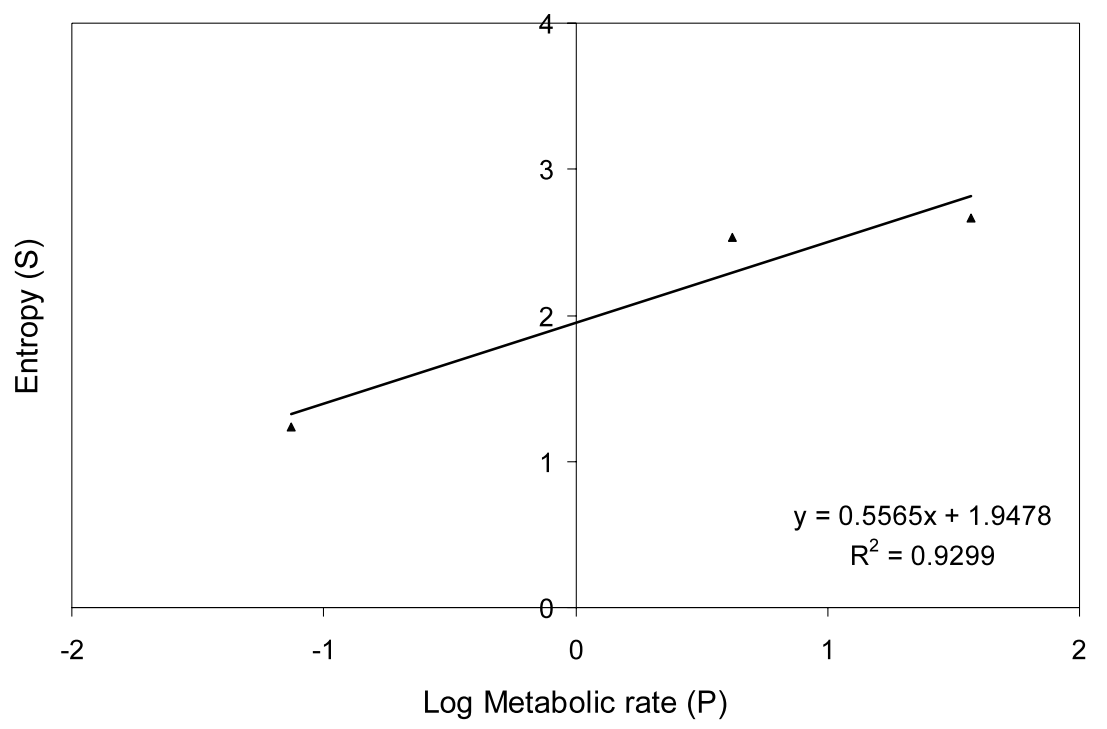

(a)

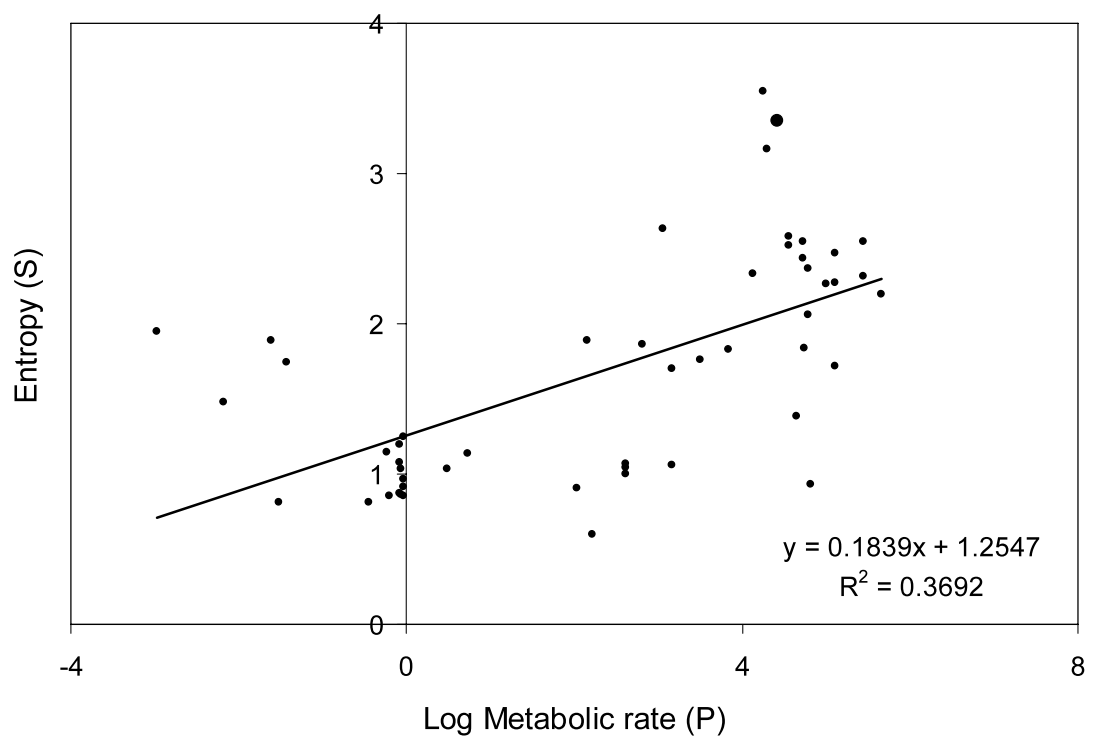

(b)

Fig. 4 The relation of logarithm of metabolic rate $(P$ in $\mathrm{W})$ to demographic entropy $(S)$ with linear fit. (a) Birds data set. (b) Mammals data set (the large dot stands for Homo sapiens).

Studies of this dependency of the rate and turnover time of metabolic processes on body size have lead to a series of empirical relations, the allometric scaling laws (7a), (7b), and $(7 \mathrm{c})$. 


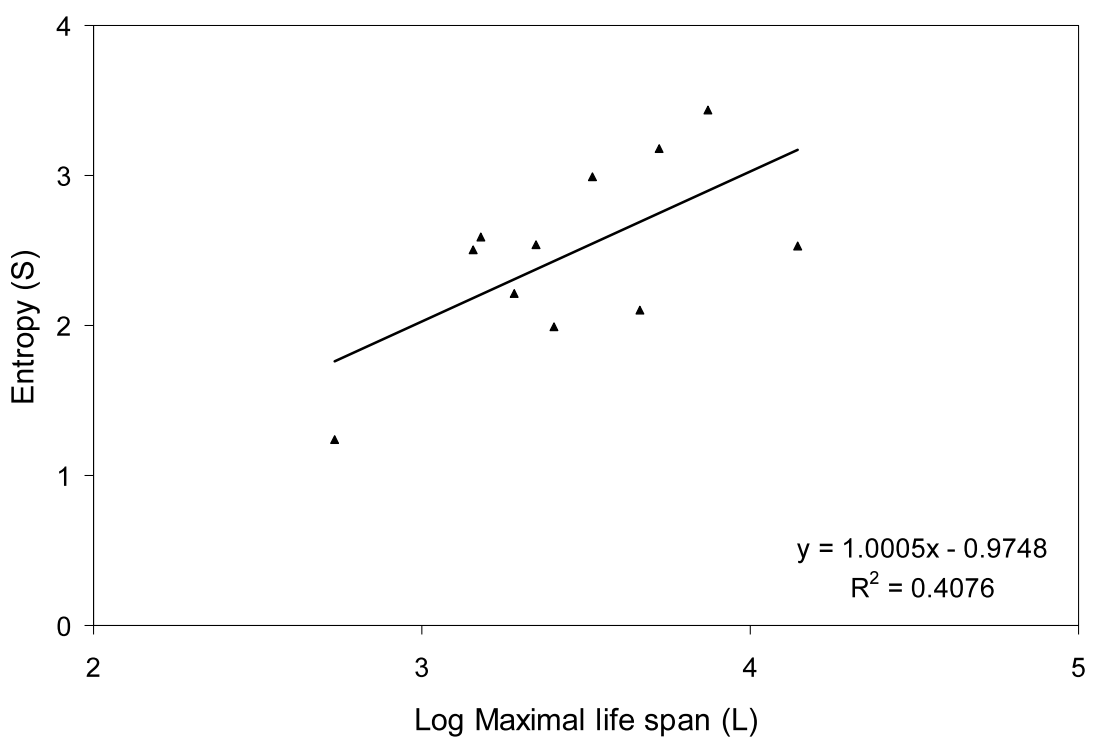

(a)

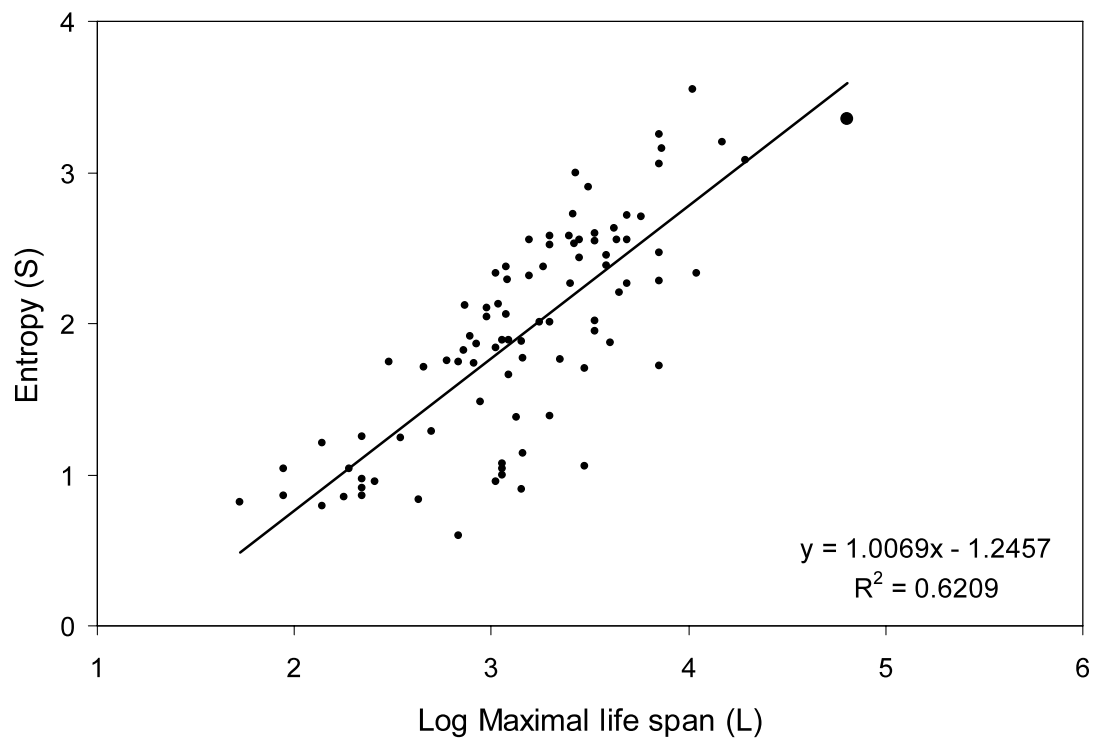

(b)

Fig. 5 The relation of logarithm of maximal life span $(L)$ to demographic entropy $(S)$ with linear fit. (a) Birds data set ( $L$ in years). (b) Mammals data set (the large dot stands for Homo sapiens) ( $L$ in years). (c) Insects data set ( $L$ in days). 


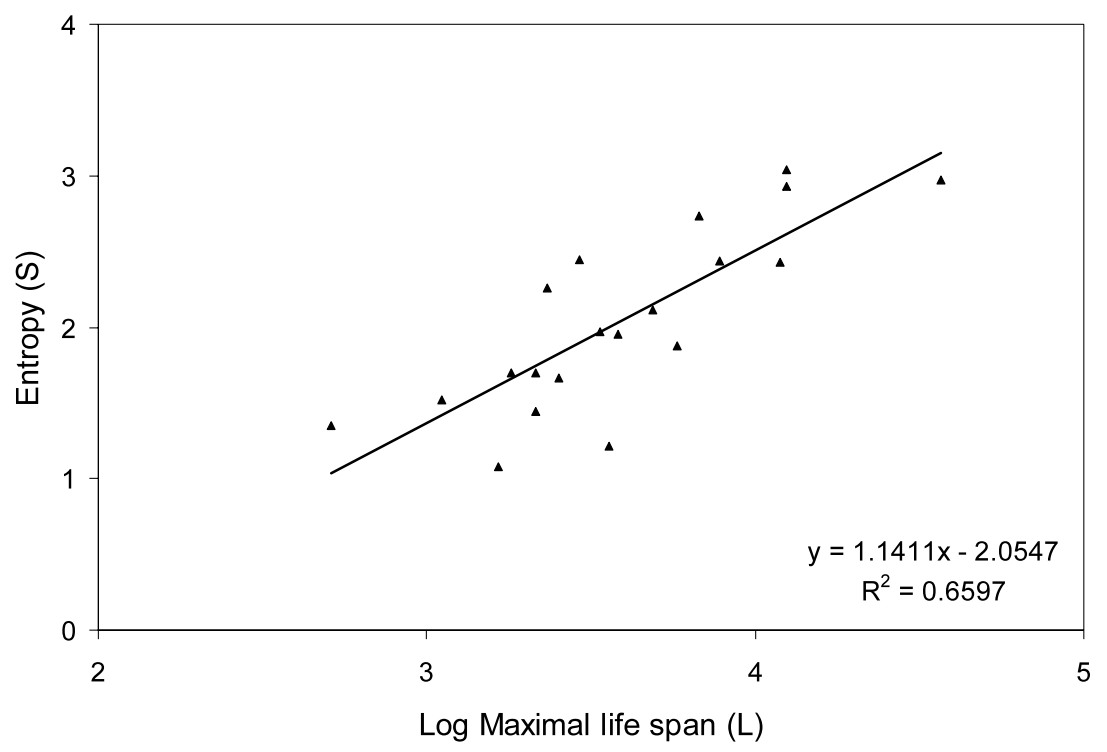

(c)

Fig. 5 Continued.

The molecular processes which underlie the scaling rules depend on the dynamics of energy transduction in biomembranes. Quantum metabolism has invoked the dynamics of electrons and protons within the membranes to provide a molecular explanation of these scaling rules. This molecular model, however, does not provide any understanding regarding the ecological and evolutionary significance of these relations.

Evidence of the ecological and evolutionary characteristics of these morphometric and physiological properties is given by their large interspecific variation. Size has been characterized in terms of certain temporal and spatial ecotypic patterns, namely: Cope's rule, the increase in size within certain phyletic lineages (Cope, 1886), Bergmann's rule, the trends in body size within species across latitude (Bergmann, 1847), and the island rule, changes in body size as species migrate from mainland to islands (Lomolino, 1985).

Metabolic rate and life span show comparable variation and are described by their unique ecotypic patterns. The magnitude of the interspecific variation in life span is highly dependent on the taxon: in insects observed life span varies 5,000 fold, in mammals 100 fold, whereas in birds 15 fold. There also exist systematic differences in life span between birds and mammals: birds are much longer lived than mammals of the same body size.

Although the form of the scaling rule relating metabolic rate and body size is universal, there are systematic differences in the scaling exponents. The scaling exponent ranges from $2 / 3$ to 1 . In a systematic review of the literature, Glazier (2005) has noted values of $2 / 3$ for birds and small mammals, $3 / 4$ for terrestrial mammals and isometric scaling $(\beta=1)$ for many pelagic animals. Reich et al. (2006) have observed an isometric scaling for perennial plants.

These patterns of variation in body size, metabolic rate, and maximal life span have a fundamental evolutionary basis. In Demetrius (2000, 2001), we exploited an analytically 
derived correlation between entropy and the parameters body size, metabolic rate, and life span to propose an evolutionary explanation of the interspecific range of variation of these parameters. The analytic relations (8a), (8b), and (8c) can be reformulated to express the morphometric and life-history variables in terms of entropy and certain taxon-specific constants:

$$
\begin{aligned}
W & =\exp \left[\frac{1}{1-\beta}\left(S-b_{1}\right)\right], \\
P & =\exp \left[\frac{\beta}{1-\beta}\left(S-b_{2}\right)\right], \\
L & =\exp \left(S-b_{3}\right)
\end{aligned}
$$

The relations indicate that given the taxon-specific constants $b_{1}, b_{2}$, and $b_{3}$ we can predict the variables body size, metabolic rate, and life span from the entropy and the scaling exponent. The relation (9c) is particularly pertinent: it indicates that maximal life span can be determined once the entropy $S$ is known. Now evolutionary entropy depends on age of sexual maturity, net-progeny size, and age of last reproduction. These quantities are empirically highly accessible. Equation (9c) thus provides a new method for estimating maximal life span.

The relations described by Eqs. (9a), (9b), and (9c) also have implications in evolutionary biology. These relations provide a framework for explaining the large interspecific variation in physiological and life-history variables. This feature derives from the fact that entropy is not only a demographic and ecological parameter. Entropy characterizes Darwinian fitness. The quantity $S$ determines the efficiency with which an organism acquires and transforms resources into metabolic work and net-offspring production. Accordingly, $S$ predicts the outcome of competition between a rate mutant and the incumbent type.

The dynamics of competition can be described in terms of Table 1. The relations in Table 1 pertain to the local changes in entropy as new mutants are introduced in the population and compete with the resident type for the available resources. The characterizations in Table 1 specify the nature of the resource constraints, the demographic constraints, and the outcome of competition between variant and incumbent for the resources.

Item (I) in Table 1 asserts that when resources are constant and limited variants with an increased entropy, in view of their increased robustness, will be more efficient in acquiring and converting resources into net-offspring production. Consequently, these variants will prevail in competition with the wild type.

Item (II) distinguishes between large and small population sizes. This distinction is relevant because when resource conditions are variable, the population size will not remain constant, but will be subject to large fluctuations. Item II(a) states that when the population size is large, since resources vary in abundance, variants with decreased entropy, and concomitantly decreased robustness, will now be more efficient in resource acquisition and utilization. Accordingly these variants will have a selective advantage. The condition II(b) concerns the case of small population size-a situation where stochastic behavior is dominant and invasion and extinction of the variant will be dependent on population size.

The significance of Eqs. (9a), (9b), and (9c) in explaining inter-specific variation in life-history patterns derives from directionality theory (Demetrius, 2000; Kowald and Demetrius, 2005; Ziehe and Demetrius, 2005), an analytic model of the evolutionary 
Table 1 Invasion criteria for a mutant allele. The quantity $\Delta S=S^{*}-S$ is the entropy differential, with $S$ and $S^{*}$ the entropy of the incumbent and the variant type, respectively. The quantity $N$ denotes population size

\begin{tabular}{lll}
$\begin{array}{l}\text { Ecological } \\
\text { constraints }\end{array}$ & $\begin{array}{l}\text { Demographic } \\
\text { condition }\end{array}$ & $\begin{array}{l}\text { Selection } \\
\text { outcome }\end{array}$ \\
\hline $\begin{array}{l}\text { (I) Constant but limited resource } \\
\text { condition }\end{array}$ & $\begin{array}{l}\Delta S>0 \\
\Delta S<0\end{array}$ & $\begin{array}{l}\text { Mutant invades almost surely (a.s.). } \\
\text { Mutant becomes extinct a.s. }\end{array}$ \\
$\begin{array}{l}\text { (II) Variable resource condition } \\
\text { (a) Large population size }\end{array}$ & $\Delta S<0$ & $\begin{array}{l}\text { Mutant invades a.s. } \\
\text { Mutant becomes extinct a.s. } \\
\text { (b) Small population size }\end{array}$ \\
& $\Delta S>0$ & $\begin{array}{l}\text { Mutant invades with a probability } \\
\text { increasing with } N .\end{array}$ \\
& $\Delta S>0$ & $\begin{array}{l}\text { Mutant becomes extinct with a } \\
\text { probability increasing with } N .\end{array}$ \\
\hline
\end{tabular}

process based on entropy as a measure of Darwinian fitness. Directionality theory distinguishes between two classes of populations: equilibrium and opportunistic species. Equilibrium species describe populations which spend the greatest part of their evolutionary history in the stationary growth phase or with a size which undergoes small fluctuations around some constant size. Opportunistic species describe populations which are subject to large irregular fluctuations in population size.

Directionality theory analyzes the global changes in evolutionary entropy which derive from two concurrent events:

(i) An invasion process - the competition between the resident population and the variants introduced by mutation.

(ii) An establishment process- the competition between the genotypes generated by interaction according to the Mendelian laws between the resident population and the new mutant.

The invasion process unfolds over a short time scale whose possible outcomes are described by Table 1 . The establishment process occurs on a comparatively long time scale and is regulated by the changes in ecological constraints which prevail under the different resource demands of the different genotypes.

According to directionality theory, the long term or global changes in evolutionary entropy which occur as the system evolves from one steady state condition to the next is contingent on the equilibrium-opportunistic distinction. The global changes in entropy within a single evolving lineage can be described by the following principles (Demetrius, 1997; Demetrius and Ziehe, 2007):

(I) Equilibrium species: a unidirectional increase in entropy.

(II) Opportunistic species:

- Large population size: a unidirectional decrease in entropy,

- Small population size: random non directional changes in entropy.

The directionality principles for entropy, when integrated with Eqs. (9a), (9b), and (9c), entail that changes in the life-history variables will be subject to evolutionary trends similar to the patterns that characterize entropy. Accordingly, our analysis provides evo- 
lutionary explanations for the ecotypic patterns in body size, metabolic rate, and life span documented in the literature; see, for example, Demetrius (2000, 2001).

\section{Appendix}

\section{Life-history data of insect populations}

Table A.1 Life-history parameters for 20 insect populations on a daily basis $(\alpha, \beta$ : age at first and last reproduction; $L$ : observed maximal life span; $T$ : generation time; $S$ : entropy)

\begin{tabular}{|c|c|c|c|c|c|c|}
\hline Species & $\alpha$ & $\beta$ & $L$ & $T$ & $S$ & Ref. \\
\hline \multicolumn{7}{|l|}{ Diptera } \\
\hline Drosophila melanogaster & 10 & 14 & 15 & 12.34 & 1.348 & $\begin{array}{l}\text { Thomas-Orillard and Legendre } \\
\text { (1996) }\end{array}$ \\
\hline Aphidoletes aphidimyza & 17 & 33 & 34 & 19.25 & 1.969 & Krivan and Havelka (2000) \\
\hline Liriomyza bryoniae & 21 & 34 & 36 & 24.29 & 1.953 & Tokumaru and Abe (2003) \\
\hline Liriomyza trifolii & 19 & 38 & 49 & 23.53 & 2.434 & Tokumaru and Abe (2003) \\
\hline Liriomyza sativae & 19 & 50 & 59 & 22.93 & 2.432 & Tokumaru and Abe (2003) \\
\hline \multicolumn{7}{|l|}{ Heteroptera } \\
\hline Orius niger $26^{\circ}$ & 20 & 46 & 46 & 25.86 & 2.738 & Baniameri et al. (2005) \\
\hline Orius niger $29^{\circ}$ & 16 & 32 & 32 & 20.72 & 2.445 & Baniameri et al. (2005) \\
\hline Orius niger $32^{\circ}$ & 16 & 29 & 29 & 19.77 & 2.257 & Baniameri et al. (2005) \\
\hline \multicolumn{7}{|l|}{ Hymenoptera } \\
\hline Aphidius gifuensis $20^{\circ}$ & 14 & 27 & 30 & 15.5 & 1.666 & Ohta and Ohtaishi (2004) \\
\hline Aphidius gifuensis $25^{\circ}$ & 11 & 24 & 28 & 12.1 & 1.443 & Ohta and Ohtaishi (2004) \\
\hline Gonatocerus ashmeadi $24^{\circ}$ & 14 & 30 & 35 & 14.96 & 1.218 & Chen et al. (2006) \\
\hline Gonatocerus ashmeadi $28^{\circ}$ & 10 & 21 & 25 & 10.63 & 1.077 & Chen et al. (2006) \\
\hline Muscidifurax raptor $25^{\circ}$ & 17 & 33 & 40 & 20.01 & 2.117 & Lysyk (2000) \\
\hline Muscidifurax raptor $30^{\circ}$ & 14 & 23 & 28 & 15.89 & 1.698 & Lysyk (2000) \\
\hline Muscidifurax raptor $33^{\circ}$ & 14 & 23 & 26 & 15.76 & 1.701 & Lysyk (2000) \\
\hline \multicolumn{7}{|l|}{ Neuroptera } \\
\hline Chrysoperla carnea & 25 & 69 & 96 & 32.28 & 2.973 & Rezaei et al. (2007) \\
\hline Micromus angulatus $25^{\circ}$ & 20 & 56 & 60 & 29.18 & 3.041 & Sato and Takada (2004) \\
\hline Micromus linearis $25^{\circ}$ & 27 & 53 & 60 & 35.44 & 2.929 & Sato and Takada (2004) \\
\hline \multicolumn{7}{|l|}{ Homoptera } \\
\hline Myzus persicae $25^{\circ}$ & 6 & 32 & 43 & 8.155 & 1.874 & Ohta and Ohtaishi (2002) \\
\hline Myzus persicae $30^{\circ}$ & 6 & 16 & 21 & 8.06 & 1.517 & Ohta and Ohtaishi (2002) \\
\hline
\end{tabular}

Open Access This article is distributed under the terms of the Creative Commons Attribution Noncommercial License which permits any noncommercial use, distribution, and reproduction in any medium, provided the original author(s) and source are credited. 


\section{References}

Baniameri, V., Soleiman-Nejadian, E., Mohaghegh, J., 2005. Life table and age dependent reproduction of the predatory bug. Orius niger Wolff (Heteroptera: Antochoridae) at three constant temperatures: a demographic analysis. Appl. Entomol. Zool. 40, 545-550.

Bergmann, C., 1847. Über die Verhaltnisse der Wärmekönomie die Tiere zu ihrer Grösse. Gött. Stud. 1, 595-708.

Bonner, J.T., 1988. The Evolution of Complexity. Princeton University Press, Princeton.

Calder, W.A., 1984. Size, Function and Life History. Harvard University Press, Cambridge.

Chen, W.L., Leopold, R.A., Morgan, D.J.W., Harris, M.O., 2006. Development and reproduction of the egg parasitoid Gonatocerus ashmeadi Girault (Hymenoptera: Mymaridae), as a function of temperature. Environ. Entomol. 35, 1178-1187.

Cope, E.D., 1886. Primary Factors of Organic Evolution. Chicago University Press, Chicago.

Demetrius, L., 1974. Demographic parameters and natural selection. Proc. Natl. Acad. Sci. U.S.A. 71, 4645-4647.

Demetrius, L., 1977. Measures of fitness and demographic stability. Proc. Natl. Acad. Sci. U.S.A. 74, 384-386.

Demetrius, L., 1983. Statistical mechanics and population biology. J. Stat. Phys. 30, 709-753.

Demetrius, L., 1997. Directionality principles in thermodynamics and evolution. Proc. Natl. Acad. Sci. U.S.A. 94, 3491-3498.

Demetrius, L., 2000. Directionality theory and the evolution of body size. Proc. R. Soc. Lond. B 267, 2385-2391.

Demetrius, L., 2001. Mortality plateaus and directionality theory. Proc. R. Soc. Lond. B 268, 2029-2037.

Demetrius, L., 2006. The origin of allometric scaling laws in biology. J. Theor. Biol. 243, 455-467.

Demetrius, L., Ziehe, M., 2007. Darwinian fitness. Theor. Popul. Biol. 72, 323-345.

Demetrius, L., Gundlach, M., Ochs, G., 2004. Complexity and demographic stability. Theor. Popul. Biol. 65, 211-215.

Dodds, P.S., Rothman, D.H., Weitz, J.S., 2001. Re-examination of the "3/4 law" of metabolism. J. Theor. Biol. 209, 9-27.

Glazier, D.S., 2005. Beyond the "3/4-power law": variation in the intra- and interspecific scaling of metabolic rate in animals. Biol. Rev. 80, 611-662.

Kleiber, M., 1961. The Fire of Life. An Introduction to Animal Energetics. Wiley, New York.

Kowald, A., Demetrius, L., 2005. Directionality theory: A computational study of an entropic principle in evolutionary processes. Proc. R. Soc. Lond. B 272, 741-749.

Krivan, V., Havelka, J., 2000. Leslie model for predatory gall-midge population. Ecol. Model. 126, 73-77.

Legendre, S., Clobert, J., Møller, A.P., Sorci, G., 1999. Demographic stochasticity and social mating system in the process of extinction of small populations: The case of passerines introduced to New Zealand. Am. Nat. 153, 449-463.

Lomolino, M.V., 1985. Body size of mammals on islands: The island rule reexamined. Am. Nat. 125, 310-316.

Lysyk, J.T., 2000. Relationships between temperature and life history parameters of Muscidifurax raptor (Hymenoptera: Pteromalidae). Environ. Entomol. 29, 596-605.

Magalhães, J.P., Costa, J., Church, G.M., 2006. An analysis of the relation between metabolism, developmental schedules, and longevity using phylogenetic independent contrasts. J. Gerontol. A Biol. Sci. Med. Sci. 62, 149-160.

Mc Kechnie, A.E., Wolf, B.O., 2004. The allometry of avian basal metabolic rate: good predictions need good data. Physiol. Biochem. Zool. 77, 502-521.

Niel, C., Lebreton, J.-D., 2005. Using demographic invariants to detect overharvested bird populations from incomplete data. Conserv. Biol. 19, 826-835.

Ohta, I., Ohtaishi., M., 2002. Life-history parameters of Myzus persicae (Sulzer) (Homoptera: Aphididae) reared on Qing-geng-cai at four constant temperatures. Jpn. J. Appl. Entomol. Zool. 46, 259-261.

Ohta, I., Ohtaishi, M., 2004. Fertility, longevity and intrinsic rate of increase of Aphidus gifuensis Ashmead (Hymenoptera: Braconidae) on the green peach aphid, Myzus persicae (Sulzer) (Homoptera: Aphididae). Appl. Entomol. Zool. 39, 113-117.

Oli, M.K., Dobson, F.S., 2003. The relative importance of life-history variables to population growth rate in mammals: Cole's prediction revisited. Am. Nat. 161, 422-440.

Peters, R.H., 1983. The Ecological Implication of Body Size. Cambridge University Press, Cambridge. 
Reich, P.B., Tjoelker, M.G., Machado, J.-L., Oleksyn, J., 2006. Universal scaling of respiratory metabolism, size and nitrogen in plants. Nature 439, 457-461.

Rezaei, M., Talebi, K., Naveh, V.H., Kavousi, A., 2007. Impacts of the pesticides imidacloprid, propargite, and pymetrozine on Chrysoperla carnea (Stephens) (Neuroptera: Chrysopidae): IOBC and life table assay. BioControl 52, 385-398.

Sato, T., Takada, H., 2004. Biological studies on three Micromus species in Japan (Neuroptera: Hemerobiidae) to evaluate their potential as biological control agents against aphids: 1 . Thermal effects on development and reproduction. Appl. Entomol. Zool. 39, 417-425.

Savage, V.M., Gillooly, J.F., Woodruff, W.H., West, G.B., Allen, A.P., Enquist, B.J., Brown, J.H., 2004. The predominance of quarter-power scaling in ecology. Funct. Ecol. 18, 257-282.

Smith, J.D.H., 2004. A macroscopic approach to demography. J. Math. Biol. 48, 105-118.

Thomas-Orillard, M., Legendre, S., 1996. Virus C de la Drosophile et dynamique d'une population hôte. C.R. Acad. Sci. Paris 319, 615-621.

Tokumaru, S., Abe, Y., 2003. Effects of temperature and photoperiod on development and reproductive potential of Liriomyza sativae, L. trifolii and L. bryoniae (Diptera: Agromyzidae), Jpn. J. Appl. Entomol. Zool. 47, 143-152.

Ziehe, M., Demetrius, L., 2005. Directionality theory: an empirical study of an entropic principle in lifehistory evolution. Proc. R. Soc. Lond. B 272, 1185-1194. 